\title{
Un engagement exceptionnel pour la formation postgraduée
}

\author{
Werner Bauera, Raphael Stolz ${ }^{b}$, Nadja Jenni ${ }^{c}$ \\ a Dr, président de I'Institut suisse pour la formation médicale postgraduée et continue, ISFM; ' ${ }^{b}$ r, vice-président de I'Institut suisse pour la formation
} médicale postgraduée et continue, ISFM; ${ }^{c}$ M.Sc., collaboratrice scientifique FMH/ISFM

L'un de vos anciens formateurs a-t-il fait preuve d'un engagement exemplaire au cours de votre formation postgraduée? Ses compétences didactiques particulières vous ont-elles permis de réaliser des progrès réjouissants? Alors n'hésitez pas à le nommer pour l'ISFM Award et à récompenser ainsi son engagement exceptionnel en faveur de la formation postgraduée des médecins.

Nous avons le plaisir de publier pour la quatrième fois la mise au concours de l'ISFM Award. Les nombreuses personnes nommées et l'écho positif que nous avons reçu confirment la pertinence et le bien-fondé de cette récompense. La remise de ce prix est devenue un événement fixe dans l'agenda de l'ISFM.

La responsabilité que portent les médecins-cadres en matière de formation postgraduée constitue un des principes fondamentaux du transfert de connaissances et de compétences aux jeunes médecins. Or cette tâche ne peut guère être définie par le seul cahier des charges, l'engagement personnel et l'enthousiasme jouent un rôle bien plus important. Dans le domaine médical, les charges qui pèsent sur les médecins sont nombreuses et les ressources en matière de temps et de moyens à disposition se réduisent sans cesse. Il est donc important que les formateurs particulièrement actifs et motivés soient reconnus. C'est pourquoi l'ISFM entend donner la possibilité aux jeunes médecins de témoigner leur reconnaissance aux formateurs qui se distinguent par leur engagement extraordinaire, sans pour autant établir un classement.

\section{Nomination par les ancien(ne)s méde- cins-assistant(e)s}

Pour être nommés à l'ISFM Award, les médecins doivent participer activement à la formation médicale postgraduée. Il s'agit notamment de médecins-cadres engagés personnellement en faveur de la formation des futurs spécialistes, considérés comme particulièrement compétents et faisant preuve d'initiative dans la manière de transmettre les connaissances et aptitudes. Pour nommer un confrère, il faut être médecin en forma- tion postgraduée ou avoir obtenu un titre de spécialiste il y a moins d'un an. Pour qu'une nomination soit valable, elle doit être déposée conjointement par deux personnes et exprimer une reconnaissance personnelle pour la qualité de la formation dispensée par le formateur et pour son engagement. Afin qu'il ne résulte ni avantage ni conflit en raison du processus de nomination, vous ne pouvez nommer que les responsables de la formation postgraduée chez lesquels vous ne travaillez plus. Les noms des personnes qui ont déposé une nomination ne seront ni publiés, ni communiqués aux nominés. Aucun classement des personnes nommées ne sera établi.

\section{Déposez votre nomination sans attendre!}

Pour nommer quelqu'un, vous pouvez télécharger le formulaire prévu à cet effet sur www.siwf.ch $\rightarrow$ ISFM $\rightarrow$

\section{Nommez sans attendre des respon- sables de la formation!}

L'ISFM Award permet d'exprimer une reconnaissance appuyée aux responsables de la formation postgraduée particulièrement engagés et compétents. Un ancien formateur vous a-t-il laissé une impression durable? Alors nommez-le pour I'ISFM Award. Nous vous prions de renvoyer le formulaire de nomination sous forme électronique à l'adresse siwf[at]fmh.ch avec la mention «ISFM Award - pour un engagement exceptionnel en faveur de la formation postgraduée».

Délai d'envoi: 6 août 2017

Vous trouverez d'autres informations sur www.siwf.ch. Si vous avez des questions, adressez-vous à siwf[at]fmh.ch ou au 031 3591111. 
Correspondance:

Institut suisse pour la for-

mation médicale postgra-

duée et continue

FMH

Elfenstrasse 18

Case postale 300

CH-3000 Berne 15

Tél. 0313591111

siwf[at]fmh.ch
Formation postgraduée $\rightarrow$ Généralités $\rightarrow$ ISFM Award, et le renvoyer dûment rempli d'ici le 6 août 2017 sous forme électronique à l'adresse suivante: siwf[at]fmh.ch La direction de l'ISFM contrôlera si la nomination est correcte du point de vue formel et validera ensuite chaque nomination.

Tous les médecins correctement nommés recevront un acte de reconnaissance et un cadeau en récompense de leur engagement pour la formation postgraduée. Ils seront cités nommément (après accord) sur le site web de l'ISFM (www.siwf.ch) et lors du Symposium MedEd du 20 septembre 2017.
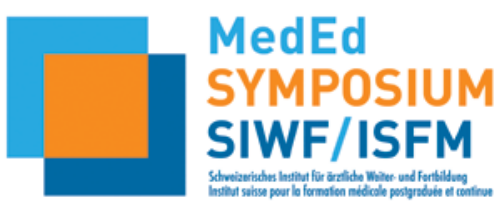

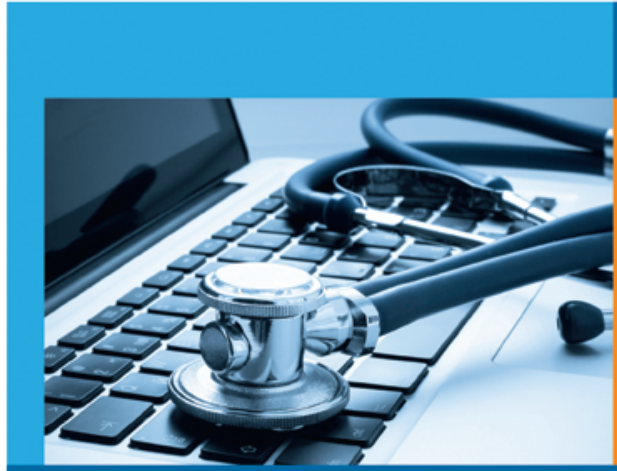

SIWF ISFM

Mit Simultanübersetzung Avec traduction simultanée

Perspektiven der ärztlichen Bildung Perspectives de la formation médicale

\section{Save the Date \\ 20. September 2017 Zentrum Paul Klee, Bern \\ Das MedEd-Symposium ist im Rahmen der erweiterten Fortbildun Le Symposium MedEd donne droit a 7 crédits dans toutes les disciplines}

